\title{
Comment on ,On the integrability of 2D Hamiltonian systems with variable Gaussian curvature" by A. A. Elmandouh
}

\author{
Wojciech Szumiński • Andrzej J. Maciejewski $(\mathbb{D}$
}

Received: 12 June 2020 / Accepted: 23 November 2020 / Published online: 22 March 2021

(C) The Author(s) 2021

\begin{abstract}
In the paper [1], the author formulates in Theorem 2 necessary conditions for integrability of a certain class of Hamiltonian systems with non-constant Gaussian curvature, which depends on local coordinates. We give a counterexample to show that this theorem is not correct in general. This contradiction is explained in some extent. However, the main result of this note is our theorem that gives new simple and easy to check necessary conditions to integrability of the system considered in [1]. We present several examples, which show that the obtained conditions are effective. Moreover, we justify that our criterion can be extended to wider class of systems, which are given by nonmeromorphic Hamiltonian functions.
\end{abstract}

Keywords Ordinary differential equations · Integrability $\cdot$ Non-integrability $\cdot$ Hamiltonian systems in cured spaces - Differential Galois theory

The research has been partially supported by the National Science Centre of Poland under Grant No. DEC-2016/21/N /ST1/02477 and by the Ministry of Science and Higher Education of Poland under the scholarship for young scientists.

W. Szumiński $(\varangle)$

Institute of Physics, University of Zielona Góra, Licealna

9, PL-65-407 Zielona Góra, Poland

e-mail: w.szuminski@if.uz.zgora.pl

A. J. Maciejewski

Janusz Gil Institute of Astronomy, University of Zielona

Góra, Licealna 9, PL-65-407 Zielona Góra, Poland

e-mail: a.maciejewski@ia.uz.zgora.pl

\section{Introduction}

The integrability and solvability studies of Hamiltonian systems in curved spaces are currently in a great activity. Let us mention papers concerning geodesic flows in various metrics $g_{i j}$ to $g_{i j}$ on manifolds governed by Hamiltonian functions $H=g^{i j} p_{i} p_{j}$, see, e.g., [2-7]. Such systems describing trajectories along geodesics are very important for understanding geometry of manifolds. Topological obstructions for their integrability can be found in [8]. Recently, geodesic flows are also very popular by their physical meaning as trajectories of test mass points in various space-times, see, e.g., $[9,10]$. Detection of gravity waves is related to the description of time-evolution of the space-distance between two mass-points on geodesics. This problem for the Zipoy-Voorhees metric around rotating Kerr black hole was investigated, e.g., in [11].

There is also a growing interest of integrability analysis of Hamiltonian systems in curved spaces with nonzero potentials. For instance, Yehia in $[12,13]$ obtained local forms of certain classes of systems with first integrals up to the fourth degree in the momenta. Valent in $[14,15]$ using the methods introduced in $[16,17]$, studied their global properties by obtaining systems defined on manifolds $\mathbb{E}^{2}, \mathbb{S}^{2}, \mathbb{H}^{2}$. Ballesteros and others $[18,19]$ constructed analogues of the harmonic oscillator and the Hénon-Heiles model in spaces with a constant curvature. The integrability of the Hamiltonian which describes the motion of a material point on a surface with a constant Gaussian curvature 
was also preformed in [20], and later enhanced in [21] to a more general form of the metric.

In the recent paper [1], the author extend the integrability analysis of Hamiltonian systems in curved spaces by a family of weight-homogeneous ones governed by the following Hamiltonian function:

$$
\left\{\begin{array}{l}
H=T+V, \\
T=\frac{1}{2} r^{n} \Lambda(\vartheta)\left(p_{r}^{2}+\frac{p_{\vartheta}^{2}}{r^{2}}\right), \\
V=r^{m} U(\vartheta),
\end{array}\right.
$$

where $n, m \in \mathbb{Z}$ and $\Lambda(\vartheta), U(\vartheta)$ are meromorphic functions. Hamiltonian (1) defines the motion of a particle moving under the influence of the homogeneous potential $V(r, \vartheta)=r^{m} U(\vartheta)$ on a surface with Gaussian curvature

$\kappa(r, \vartheta)=\frac{r^{n-2}}{\Lambda(\vartheta)}\left[\Lambda(\vartheta) \Lambda^{\prime \prime}(\vartheta)-\Lambda^{\prime}(\vartheta)^{2}\right]$,

where prime denotes the derivative with respect to $\vartheta$.

The author of paper [1] derived the necessary integrability conditions of system (1) deduced from the Morales-Ramis theory [22]. This approach is based on the analysis of the differential Galois group of variational equations of a considered system along a certain particular solution. The Morales-Ramis theory has been used recently for study integrability of various important physical and astronomical systems, see, e.g., papers [23-33]. We also mention review paper [34], in which certain examples can be found.

In most applications, the variational equations are transformed into a system with rational coefficients and, if it, or its subsystem, can be written as a one second-order equation, then the verification if the identity component of its differential Galois group is Abelian can be performed with the help of the Kovacic algorithm [35]. However, sometimes it is possible to transform the variational equations into the system of differential equations with known differential Galois group, for instance into the Riemann $P$-equation.

This is the case of [1]. The author calculated the variational equations of Hamilton's equations governed by Hamiltonian (1) along a particular solution lying on the manifold

$$
\mathscr{N}=\left\{\left(r, p_{r}, \vartheta, p_{\vartheta}\right) \in \mathbb{C}^{4} \mid \vartheta=\vartheta_{0}, p_{\vartheta}=0\right\} \text {. }
$$

Next, thanks to the change of the independent variable

$t \rightarrow z=\frac{U\left(\vartheta_{0}\right)}{h} r^{m}$, with $m h U\left(\vartheta_{0}\right) \neq 0$, where $h$ is the energy of the chosen particular solutions, the author transformed the variational equations into the Riemann $P$-equation

$$
\begin{aligned}
& \Theta^{\prime \prime}(z)+\left(\frac{1-\alpha-\alpha^{\prime}}{z}+\frac{1-\beta-\beta^{\prime}}{z-1}\right) \Theta^{\prime}(z) \\
& +\left(\frac{\alpha \alpha^{\prime}}{z^{2}}+\frac{\beta \beta^{\prime}}{(z-1)^{2}}+\frac{\gamma \gamma^{\prime}-\alpha \alpha^{\prime}-\beta \beta^{\prime}}{z(z-1)}\right) \Theta(z)=0,
\end{aligned}
$$

where $\Theta$ denotes the variation of $\vartheta$. Then, he introduced the new parameters

$\lambda_{1}:=m^{2} \frac{\Lambda^{\prime \prime}\left(\vartheta_{0}\right)}{\Lambda\left(\vartheta_{0}\right)}, \quad \lambda_{2}:=1+\frac{U^{\prime \prime}\left(\vartheta_{0}\right)}{(m-n) U\left(\vartheta_{0}\right)}$,

and he wrote the differences of the exponents at the singularities $z=0, z=1, z=\infty$, in the form

$$
\begin{aligned}
& \rho=\alpha-\alpha^{\prime}=\frac{\sqrt{(n-2)^{2}-8 \lambda_{1}}}{2 m}, \\
& \sigma=\beta-\beta^{\prime}=\frac{1}{2}, \\
& \tau=\gamma-\gamma^{\prime}=\frac{\sqrt{(m-n-2)^{2}+8(m-n) \lambda_{2}-8 \lambda_{1}}}{2 m} .
\end{aligned}
$$

The author of [1] derived the necessary integrability conditions of system (1) using the Kimura theorem [36], see also Appendix. This theorem gives the necessary and sufficient conditions, which guarantee that the identity component of the Riemann $P$-equation is solvable. The main result of paper [1] is the following theorem.

Theorem 1 (Elmandouh [1]) Suppose the two functions $\Lambda(\vartheta)$ and $U(\vartheta)$ are two meromorphic functions, and assume there is $\vartheta_{0} \in \mathbb{C}$ such that

$\Lambda^{\prime}\left(\vartheta_{0}\right)=0=U^{\prime}\left(\vartheta_{0}\right)$, with $\Lambda\left(\vartheta_{0}\right) U\left(\vartheta_{0}\right) \neq 0$.

If the Hamiltonian system (1) is Liouville integrable, then the number

$$
\begin{aligned}
\lambda & :=\lambda_{2}-\frac{\lambda_{1}}{m-n} \\
& =1+\frac{1}{m-n}\left[\frac{U^{\prime \prime}\left(\vartheta_{0}\right)}{U\left(\vartheta_{0}\right)}-m^{2} \frac{\Lambda^{\prime \prime}\left(\vartheta_{0}\right)}{\Lambda\left(\vartheta_{0}\right)}\right],
\end{aligned}
$$

belongs to the sets $\mathscr{J}_{i}(m, n)$, which are listed in Table 1.

We claim that this theorem is in general not correct. Let us exemplify this by the Hamiltonian function

$$
H=\frac{1}{2 \sin ^{2} \vartheta} r^{6}\left(p_{r}^{2}+\frac{p_{\vartheta}^{2}}{r^{2}}\right)+\left(\frac{r}{\sin \vartheta}\right)^{m},
$$


where $m \in \mathbb{Z}^{*}$. For this Hamiltonian, we have

$n=6, \quad \Lambda(\vartheta)=\frac{1}{\sin ^{2} \vartheta}, \quad U(\vartheta)=\frac{1}{\sin ^{m} \vartheta}$.

Both $\Lambda$ and $U$ are meromorphic functions so the first statement of Theorem 1 is satisfied.

We take point $\vartheta_{0}=\frac{\pi}{2}$ at which condition (8) is fulfilled. Then, value of $\lambda$ defined in (9) at $\vartheta_{0}$ is

$\lambda=1+\frac{m}{m-6}(1-2 m)$.

Comparing this value with the forms of the sets $\mathscr{J}_{i}$ defined in Table 1 in [1], we conclude that only for $m=$ \pm 1 , the necessary integrability condition is satisfied. It is even better visible when we look at the differences of the exponents (7). In this case they took the values

$\rho=\frac{2 \sqrt{1-m^{2}}}{m}, \quad \sigma=\frac{1}{2}, \quad \tau=\frac{\sqrt{16-15 m^{2}}}{2 m}$.

Now it is evident that for $m \neq \pm 1$ neither case $A$ nor case $B$ of the Kimura theorem can be fulfilled because $\rho$ and $\tau$ are both complex.

Since the necessary integrability condition is not satisfied due to $\lambda \notin \mathscr{J}_{i}$, then, according to Theorem 1, it means that for $m \neq \pm 1$ system (10) is not integrable. However, this conclusion is actually wrong because Hamiltonian (10) possesses the additional first integral for arbitrary $m$, and it has the form:

$I=r^{2} \cos \vartheta p_{r}+r \sin \vartheta p_{\vartheta}$,

see [37]. As $I$ is functionally independent with $H$ implies that system (10) is, in fact, integrable.

We discuss this contradiction in the last section of this paper. At first, we give the correct formulation of the integrability obstructions for system (1). However, we define the main integrability theorem in the form different as in [37]. It is simply, effective and easy for applications.

\section{Integrability analysis}

We state the following theorem.

Theorem 2 Assume that $\Lambda(\vartheta)$ and $U(\vartheta)$ are complex meromorphic functions of variable $\vartheta$ and there exist a point $\vartheta_{0} \in \mathbb{C}$, such that

$\Lambda^{\prime}\left(\vartheta_{0}\right)=0=U^{\prime}\left(\vartheta_{0}\right)$, with $\Lambda\left(\vartheta_{0}\right) U\left(\vartheta_{0}\right) \neq 0 .(15)$
If the Hamiltonian system defined by Hamiltonian (1) is integrable in the sense of Liouville, then the numbers

$$
\begin{aligned}
& \lambda_{1}:=\frac{1}{2 m} \sqrt{(n-2)^{2}-8 \frac{\Lambda^{\prime \prime}\left(\vartheta_{0}\right)}{\Lambda\left(\vartheta_{0}\right)},} \\
& \lambda_{2}:=\frac{1}{2 m} \sqrt{(m-n+2)^{2}+8\left(\frac{U^{\prime \prime}\left(\vartheta_{0}\right)}{U\left(\vartheta_{0}\right)}-\frac{\Lambda^{\prime \prime}\left(\vartheta_{0}\right)}{\Lambda\left(\vartheta_{0}\right)}\right)},
\end{aligned}
$$

belong to one item of the following list

$$
\begin{aligned}
& \text { Lp. } \quad \lambda_{1} \\
& \lambda_{2} \\
& \text { (i) arbitrary } \\
& \frac{1}{2}+2 p \pm \lambda_{1}, \frac{3}{2}+2 p \pm \lambda_{1}, \frac{1}{2}+p \\
& \text { (ii) } \frac{1}{2}+s \\
& \text { arbitrary } \\
& \text { (iii) } \pm \frac{1}{3}+s \quad \pm \frac{1}{3}+p, \pm \frac{1}{4}+p, \pm \frac{1}{5}+p, \pm \frac{2}{5}+p \\
& \text { (iv) } \pm \frac{1}{4}+s \quad \pm \frac{1}{3}+p \\
& \text { (v) } \pm \frac{1}{5}+s \quad \pm \frac{1}{3}+p, \pm \frac{2}{5}+p \\
& \text { (vi) } \pm \frac{2}{5}+s \\
& \pm \frac{1}{3}+p, \pm \frac{1}{5}+p
\end{aligned}
$$

where $m, n, s, p \in \mathbb{Z}$ and $m \neq 0$.

Proof Since the variational equations given in Eq. (18) in [1] are incorrectly calculated, we make the integrability analysis of system (1) starting from the very beginning.

The Hamiltonian equations $\dot{\boldsymbol{x}}=\boldsymbol{X}_{H}(\boldsymbol{x})$ governed by Hamiltonian (1) are as follows:

$$
\left\{\begin{array}{l}
\dot{r}=r^{n} \Lambda(\vartheta) p_{r}, \\
\dot{p}_{r}=-\frac{n}{2} r^{n-1} \Lambda(\vartheta) p_{r}^{2}+\frac{2-n}{2} r^{n-3} \Lambda(\vartheta) p_{\vartheta}^{2}-m r^{m-1} U(\vartheta), \\
\dot{\vartheta}=r^{n-2} \Lambda(\vartheta) p_{\vartheta}, \\
\dot{p}_{\vartheta}=-\frac{1}{2} r^{n} \Lambda^{\prime}(\vartheta)\left(p_{r}^{2}+r^{-2} p_{\vartheta}^{2}\right)-r^{m} U^{\prime}(\vartheta) .
\end{array}\right.
$$

If we assume that there exist a point $\vartheta_{0} \in \mathbb{C}$ such that $U^{\prime}\left(\vartheta_{0}\right)=0=\Lambda^{\prime}\left(\vartheta_{0}\right)$, then system (18) has two-dimensional invariant manifold (3). Restricting the right-hand sides of (18) to $\mathscr{N}$, we obtain

$$
\left\{\begin{array}{l}
\dot{r}=r^{n} \Lambda\left(\vartheta_{0}\right) p_{r}, \\
\dot{p}_{r}=-\frac{n}{2} r^{n-1} \Lambda\left(\vartheta_{0}\right) p_{r}^{2}-m r^{m-1} U\left(\vartheta_{0}\right), \\
\dot{\vartheta}=0 \\
\dot{p}_{\vartheta}=0
\end{array}\right.
$$


Solution of (19) determines our particular solution $\mathbb{C} \ni$ $t \rightarrow \varphi_{\mathscr{N}}(t)=\left(r(t), p_{r}(t), 0,0\right)$. In fact, we have the whole family of particular solutions lying on a fixed energy level

$H_{\mathscr{N}}=\frac{1}{2} r^{n} \Lambda\left(\vartheta_{0}\right) p_{r}^{2}+r^{m} U\left(\vartheta_{0}\right)=h$.

Let $\boldsymbol{y}:=\left[R, P_{R}, \Theta, P_{\Theta}\right]^{T}$ define the variations of $\boldsymbol{x}:=\left[r, p_{r}, \vartheta, p_{\vartheta}\right]^{T}$. Then, the first-order variational equations along the particular solution $\varphi_{\mathscr{N}}(t)$ are given by

$\dot{\boldsymbol{y}}=A(t) \cdot \boldsymbol{y}, \quad A(t):=\frac{\partial \boldsymbol{X}_{H}}{\partial \boldsymbol{x}}\left(\boldsymbol{\varphi}_{\mathscr{N}}(t)\right)$.

The explicit form of the Jacobian matrix $A(t)$ is as follows:

$A=\left(\begin{array}{cccc}n r^{n-1} \Lambda\left(\vartheta_{0}\right) p_{r} & r^{n} \Lambda\left(\vartheta_{0}\right) & 0 & 0 \\ a\left(r, p_{r}\right) & -n r^{n-1} \Lambda\left(\vartheta_{0}\right) p_{r} & 0 & 0 \\ 0 & 0 & 0 & r^{n-2} \Lambda\left(\vartheta_{0}\right) \\ 0 & 0 & b\left(r, p_{r}\right) & 0\end{array}\right)$,

where

$$
\begin{aligned}
& a=\frac{n(1-n)}{2} r^{n-2} \Lambda\left(\vartheta_{0}\right) p_{r}^{2}+m(1-m) r^{m-2} U\left(\vartheta_{0}\right), \\
& b=-\frac{1}{2} r^{n} \Lambda^{\prime \prime}\left(\vartheta_{0}\right) p_{r}^{2}-r^{m} U^{\prime \prime}\left(\vartheta_{0}\right) .
\end{aligned}
$$

Since the motion takes place on $\left(r, p_{r}\right)$ plane, the equations for $\dot{\Theta}$ and $\dot{P}_{\Theta}$ form a closed subsystem of normal variational equations. For simplicity, we write them as a one second-order differential equation

$\ddot{\Theta}+P\left(r, p_{r}\right) \dot{\Theta}+Q\left(r, p_{r}\right) \Theta=0$,

where the coefficients $P$ and $Q$ are given by

$$
\begin{aligned}
& P=(2-n) r^{n-1} \Lambda\left(\vartheta_{0}\right) p_{r}, \\
& Q=\frac{1}{2} r^{n-2} \Lambda\left(\vartheta_{0}\right)\left[r^{n} \Lambda^{\prime \prime}\left(\vartheta_{0}\right) p_{r}^{2}+2 r^{m} U^{\prime \prime}\left(\vartheta_{0}\right)\right] .
\end{aligned}
$$

Next, we perform the change of the independent variable (4) by using the energy first integral (20). Hence, we transform the normal variational equation (23) into the system with rational coefficients

$$
\begin{aligned}
& \Theta^{\prime \prime}(z)+\frac{1}{2}\left(\frac{1}{z-1}+\frac{2 m-n+2}{m z}\right) \Theta^{\prime}(z) \\
& +\frac{1}{2 m^{2}}\left(\frac{\Lambda^{\prime \prime}\left(\vartheta_{0}\right)}{\Lambda\left(\vartheta_{0}\right) z}-\frac{U^{\prime \prime}\left(\vartheta_{0}\right)}{U\left(\vartheta_{0}\right) z(z-1)}\right) \Theta(z)=0 .
\end{aligned}
$$

This equation is the Riemann $P$-equation (5), with the differences of the exponents at singularities $z=0, z=$ 1 and $z=\infty$ defined simply by

$\rho=\lambda_{1}, \quad \sigma=\frac{1}{2}, \quad \tau=\lambda_{2}$, where the quantities $\lambda_{1}$ and $\lambda_{2}$ are postulated in (16). The proof of Theorem 2 consists of the direct application of the Kimura theorem 3 to the obtained Riemann $P$-equation (25) with the differences of the exponents (26). Indeed, the first four elements given in item (i) of the list (17) were deduced from Case $A$ of Theorem 3 , while the remaining entries of (17) come from Case $B$ of this theorem.

Remark 1 Let us underline that in our analysis we assumed that $m \neq 0$. This is because for $m=0$ the normal variational equations (21) are solvable. Thus, in this case there is no obstacle for the integrability of the considered system. In order, to define the integrability obstructions for the system higher-order variational equations or a different approach must be used.

From the form of integrability list (17), we can deduce the following corollary.

\section{Corollary 1 Let us assume that the quantities}

$\frac{\Lambda^{\prime \prime}\left(\vartheta_{0}\right)}{\Lambda\left(\vartheta_{0}\right)} \in \mathbb{Q}, \quad \frac{U^{\prime \prime}\left(\vartheta_{0}\right)}{U\left(\vartheta_{0}\right)} \in \mathbb{Q}$.

If $\lambda_{1}$ and $\lambda_{2}$ defined in (16) are both irrational, then system (1) is not integrable in the Liouville sense.

Proof Suppose quantities (16) have the form

$\lambda_{1}=\frac{\sqrt{r}}{2 m}, \quad \lambda_{2}=\frac{\sqrt{s}}{2 m}, \quad r, s \in \mathbb{Q}$

and they are irrational. If system (1) is integrable, then according to Theorem 2, one of two possibilities

$\sqrt{s}=k+\sqrt{r}, \quad$ or $\quad \sqrt{s}=k-\sqrt{r}, \quad k \in \mathbb{Z}^{*}$,

holds true. However, squaring (29), we get

$s=k^{2}+2 k \sqrt{r}+r, \quad$ or $s=k^{2}-2 k \sqrt{r}+r$,

which is the contradiction because $r, s \in \mathbb{Q}$, while the right-hand sides of (30) are irrational. This ends the proof.

\section{Applications of Theorem 2}

In this section, we present the advantage of Theorem 2 by applying it on some simple examples. For these models, the metrics have nonzero curvature.

Example 1 As the first example, we reconsider the Hamiltonian function (10). We take point $\vartheta_{0}=\frac{\pi}{2}$ 
at which condition (15) is fulfilled. Taking this into account, we have the following

$n=6, \quad \frac{\Lambda^{\prime \prime}\left(\frac{\pi}{2}\right)}{\Lambda\left(\frac{\pi}{2}\right)}=2, \quad \frac{U^{\prime \prime}\left(\frac{\pi}{2}\right)}{U\left(\frac{\pi}{2}\right)}=m$.

Then, from definition (16) we get

$\lambda_{1}=0, \quad \lambda_{2}=\frac{1}{2} \operatorname{sgn}(m)$,

It is easy to see that these values of $\lambda_{1}$ and $\lambda_{2}$ belong to the first item (i) of integrability list (17). Thus, the necessary integrability condition is satisfied for arbitrary $m \in \mathbb{Z}^{*}$, as it should be since we have already met that Hamiltonian (10) is integrable with the first integral (14).

Example 2 We have defined $\lambda_{1}$ and $\lambda_{2}$ in such a way that they equal to the differences of the exponents at $z=0$ and $z=\infty$, see (26). With this choice checking conditions is straightforward and we avoid to derive and to check cumbersome form of sets $\mathscr{J}_{i}(m, n)$ listed in Table 1 of [1]. For instance, if $\lambda_{1}$ and $\lambda_{2}$ are both irrational and condition (27) is satisfied, then according to Corollary 1, the system is not integrable. Let us exemplify this by the following two-parameter family of Hamiltonian systems generated by the Hamiltonian function

$H=\frac{1}{2} r^{n} \cos \frac{\vartheta}{3}\left(p_{r}^{2}+\frac{p_{\vartheta}^{2}}{r^{2}}\right)+r^{m} \cos \vartheta$.

Hence, functions $\Lambda, U$ standing in (1), are as follows:

$\Lambda(\vartheta)=\cos \frac{\vartheta}{3}, \quad U(\vartheta)=\cos \vartheta$.

At point $\vartheta_{0}=0$, quantities (27) read

$\frac{\Lambda^{\prime \prime}\left(\vartheta_{0}\right)}{\Lambda\left(\vartheta_{0}\right)}=-\frac{1}{9}, \quad \frac{U^{\prime \prime}\left(\vartheta_{0}\right)}{U\left(\vartheta_{0}\right)}=-1$,

and the integrability coefficients (16) are given by

$\lambda_{1}=\frac{\sqrt{9(n-2)^{2}+8}}{6 m}$,

$\lambda_{2}=\frac{\sqrt{9(m-n+2)^{2}-64}}{6 m}$.

It can be proved by simple arguments that $\lambda_{1}$ and $\lambda_{2}$ are both irrational for all values of the parameters $n, m \in \mathbb{Z}$ with $m \neq 0$. Thus, according to Corollary 1, twoparameter family of Hamiltonian systems governed by (33) is not integrable in the sense of Liouville.
Claim 1 The next example shows that in many cases we need to modify assumptions of Theorem 2. In practice, we have to consider systems for which the Hamiltonian is not meromorphic. The best known example is the N-body problem. Nevertheless, the MoralesRamis theorem was used successfully to prove the non-integrability. The methods to cope with algebraic potentials are described in [38] and [39]. Here, we claim that in Theorem 2 we can assume that numbers $n$ and $m$ are rational. Moreover, we can assume that functions $\Lambda(\vartheta)$ and $U(\vartheta)$ are algebraic functions of $\cos (\vartheta)$ or $\sin (\vartheta)$. Then, the thesis of this theorem is valid with a small modification. To prove this we introduce additional variables in order to extend the system to a higher-dimensional space. The key point is to make this extension in such a way that the right-hand sides of the extended system are rational. For the consider system, it can be done in the following way:

1. We introduce new variables $c=\cos \vartheta$ and $s=$ $\sin \vartheta$. Of course, $f_{1}(c, s)=c^{2}+s^{2}-1=0$.

2. Then, $v(c, s)=\Lambda(\vartheta)$ and $u(c, s)=U(\vartheta)$ are algebraic functions of $(c, s)$. Let $f_{2}(v, c, s)$ and $f_{3}(u, c, s)$ be their respective minimal polynomials.

3. Finally, let $\rho_{1}=r^{n}$ and $\rho_{2}=r^{m}$ and let $f_{4}\left(\rho_{1}, r\right)$ and $f_{5}\left(\rho_{2}, r\right)$ be their respective minimal polynomials.

4. Now we take variables $\mathbf{z}=\left(r, p_{r}, p_{\vartheta}, c, s, u, v, \rho_{1}\right.$, $\rho_{2}$ ) and calculate their time derivatives using the original Hamilton's equations, standard rules of differentiation of compositions and implicit functions.

5. One can check that obtained system $\dot{\mathbf{z}}=\mathbf{Z}(\mathbf{z})$ has rational right-hand sides. Moreover, the Hamiltonian, which in new variables reads

$$
H=\frac{1}{2} \rho_{1} v\left(p_{r}^{2}+\frac{p_{\vartheta}^{2}}{r^{2}}\right)+\rho_{2} u,
$$

has first integrals $f_{1}, \ldots, f_{5}$.

6. On the common level $f_{i}(z)=0$ for $i=1, \ldots 5$, the extended system coincides with the original one.

After the described extension, we can apply the MoralesRamis theory. In practice, however, it is allowed to perform all the calculations in the original variables in the way we show in the example below. 
Example 3 We consider one-parameter family of Hamiltonian systems governed by a Hamiltonian function of the form:

$$
H=\frac{1}{2} r^{2-\frac{m}{2}} \cos ^{\frac{2}{3}}\left(\frac{3 m}{2} \vartheta\right)\left(p_{r}^{2}+\frac{p_{\vartheta}^{2}}{r^{2}}\right)+r^{m} \cos ^{\frac{2}{3}}\left(\frac{3 m}{2} \vartheta\right)
$$

We take point $\vartheta_{0}=0$ at which condition (15) is fulfilled. Taking this into account, we have the following

$n=2-\frac{m}{2}, \quad \frac{\Lambda^{\prime \prime}(0)}{\Lambda(0)}=-\frac{3 m^{2}}{2}=\frac{U^{\prime \prime}(0)}{U(0)}$.

Thus, the integrability coefficients (16) take the values

$\lambda_{1}=\frac{7}{4} \operatorname{sgn}(m), \quad \lambda_{2}=\frac{3}{4} \operatorname{sgn}(m)$.

It is easy to verify that these values of $\lambda_{1}$ and $\lambda_{2}$ belong to the first item (i) of integrability list (17). Thus, the necessary integrability condition is satisfied for arbitrary $m \in \mathbb{Q}^{*}$. Indeed, the Hamiltonian system defined by Hamiltonian (38) is integrable. The additional first integral, functionally independent with (38), is defined by

$$
\begin{aligned}
I & =r^{2-\frac{3 m}{2}} \cos \left(\frac{3 m}{2} \vartheta\right)\left(3 p_{r}^{2}+\frac{p_{\vartheta}^{2}}{r^{2}}\right) p_{\vartheta} \\
& +2 r^{3-\frac{3 m}{2}} \sin \left(\frac{3 m}{2} \vartheta\right) p_{r}^{3} \\
& +4 r \sin \left(\frac{3 m}{2} \vartheta\right) p_{r}+4 \cos \left(\frac{3 m}{2} \vartheta\right) p_{\vartheta} .
\end{aligned}
$$

According to our knowledge, this is the new integrable system with the non-constant Gaussian curvature of the form:

$\kappa(r . \vartheta)=-\frac{3 m^{2}}{2} r^{-\frac{m}{2}}\left[\cos \left(\frac{3 m}{2} \vartheta\right)\right]^{-\frac{4}{3}}$.

\section{Remarks and conclusions}

In this comment, we pointed out that Theorem 2 postulated in paper [1] is in general incorrect. We give the example of integrable system, which is according to Theorem 2 not integrable. It is rather difficult to find a unique source of failure in Theorem 2. First of all the differences of the exponents (7) of the Riemann $P$ equation (5) were incorrectly calculated. Equivalently, the parameter $\lambda_{1}$, which appears in $\rho$ and $\tau$ was wrongly defined. This implies that the forms of sets $\mathscr{J}_{i}$, given in Eq. (29) in work [1], are also wrongly defined because $\Delta_{1}=\sqrt{(n-2)^{2}-8 \lambda_{1}}$ is incorrect.

To get correct forms of the differences of exponents (7), one has to define $\lambda_{1}:=\frac{\Lambda^{\prime \prime}\left(\vartheta_{0}\right)}{\Lambda\left(\vartheta_{0}\right)}$, not as postulated in Eq. (6). This permanently repeated mistake causes Theorem 1 to be generally incorrect, i.e., it is only valid for $m= \pm 1$. Unfortunately, in its applications the author of [1] has chosen examples in such a way that all of them have $m=1$. This gave to the author as well as to the reviewers false belief in the correctness of the preformed calculations. Nonetheless, the above described redefinition of $\lambda_{1}$ does not make Theorem 1 fully correct. Moreover, the author restricts conditions given by Case $B$ of the Kimura theorem and does not explicitly worn the reader about this. For example, in the proof of Case 1 on p. 937 Eq. (31) restriction $\omega \in \mathbb{Z}$ is too strong. Condition $n \in \mathbb{Z}$ is fulfilled if and only if $\omega=l / m$ for a certain $l \in \mathbb{Z}$. The same thing holds in the remaining cases. This implies that restriction for $m$ to be a multiplicity of 2, 3, 5, see Table 1 in [1], seems to be unnecessary.

However, our aim in this note was to formulate theorem, which gives new form of the necessary integrability conditions of system (1). The key point is that we avoided complications connected with clumsy form of sets $\mathscr{J}_{i}(m, n)$. We give three, nontrivial examples which show that the presented formulation is easy and effective in applications. Moreover, we show also how we can extend our criterion to wider class of systems, which are given by non-meromorphic Hamiltonian functions.

Acknowledgements The research has been supported by the National Science Centre of Poland under Grant No. DEC2016/21/N /ST1/02477 and by the Ministry of Science and Higher Education of Poland under the scholarship for young scientists.

Conflict of interest The authors declare that the research was conducted in the absence of any conflict of interest.

Open Access This article is licensed under a Creative Commons Attribution 4.0 International License, which permits use, sharing, adaptation, distribution and reproduction in any medium or format, as long as you give appropriate credit to the original author(s) and the source, provide a link to the Creative Commons licence, and indicate if changes were made. The images or other third party material in this article are included in the article's Creative Commons licence, unless indicated otherwise in a credit line to the material. If material is not included in the article's Creative Commons licence and your intended use is not permitted by statutory regulation or exceeds the permitted use, you will need 
Table 1 Schwarz's table. Here $r, q, p \in \mathbb{Z}$

\begin{tabular}{|c|c|c|c|c|}
\hline 1 & $1 / 2+r$ & $1 / 2+q$ & arbitrary & \\
\hline 2 & $1 / 2+r$ & $1 / 3+q$ & $1 / 3+p$ & \\
\hline 3 & $2 / 3+r$ & $1 / 3+q$ & $1 / 3+p$ & $r+q+p$ even \\
\hline 4 & $1 / 2+r$ & $1 / 3+q$ & $1 / 4+p$ & \\
\hline 5 & $2 / 3+r$ & $1 / 4+q$ & $1 / 4+p$ & $r+q+p$ even \\
\hline 6 & $1 / 2+r$ & $1 / 3+q$ & $1 / 5+p$ & \\
\hline 7 & $2 / 5+r$ & $1 / 3+q$ & $1 / 3+p$ & $r+q+p$ even \\
\hline 8 & $2 / 3+r$ & $1 / 5+q$ & $1 / 5+p$ & $r+q+p$ even \\
\hline 9 & $1 / 2+r$ & $2 / 5+q$ & $1 / 5+p$ & \\
\hline 10 & $3 / 5+r$ & $1 / 3+q$ & $1 / 5+p$ & $r+q+p$ even \\
\hline 11 & $2 / 5+r$ & $2 / 5+q$ & $2 / 5+p$ & $r+q+p$ even \\
\hline 12 & $2 / 3+r$ & $1 / 3+q$ & $1 / 5+p$ & $r+q+p$ even \\
\hline 13 & $4 / 5+r$ & $1 / 5+q$ & $1 / 5+q$ & $r+q+p$ even \\
\hline 14 & $1 / 2+r$ & $2 / 5+q$ & $1 / 3+p$ & \\
\hline 15 & $3 / 5+r$ & $2 / 5+q$ & $1 / 3+p$ & $r+q+p$ even \\
\hline
\end{tabular}

to obtain permission directly from the copyright holder. To view a copy of this licence, visit http://creativecommons.org/licenses/ by/4.0/.

\section{Appendix. The Riemann $\boldsymbol{P}$-equation}

The Riemann $P$-equation is the most general secondorder differential equation with three regular singularities. If we place the singularities at $z=0, z=1$ and $z=\infty$, it is given by

$$
\begin{aligned}
& \Theta^{\prime \prime}(z)+\left(\frac{1-\alpha-\alpha^{\prime}}{z}+\frac{1-\beta-\beta^{\prime}}{z-1}\right) \Theta^{\prime}(z) \\
& +\left(\frac{\alpha \alpha^{\prime}}{z^{2}}+\frac{\beta \beta^{\prime}}{(z-1)^{2}}+\frac{\gamma \gamma^{\prime}-\alpha \alpha^{\prime}-\beta \beta^{\prime}}{z(z-1)}\right) \Theta(z)=0,
\end{aligned}
$$

where $\left(\alpha, \alpha^{\prime}\right),\left(\beta, \beta^{\prime}\right)$ and $\left(\gamma, \gamma^{\prime}\right)$ are the exponents at the respective singular points, see, for instance, Refs. $[40,41]$. These exponents satisfy the Fuchs relation

$\alpha+\alpha^{\prime}+\gamma+\gamma^{\prime}+\beta+\beta^{\prime}=1$.

We denote the differences of the exponents by

$\rho=\alpha-\alpha^{\prime}, \quad \sigma=\beta-\beta^{\prime}, \quad \tau=\gamma-\gamma^{\prime}$.

Necessary and sufficient conditions for solvability of the identity component of the differential Galois group of (43) are given by the following theorem due to Kimura [36].

Theorem 3 The identity component of the differential Galois group of Riemann P-equation (43) is solvable iff
A. at least one of the four numbers $\rho+\sigma+\tau,-\rho+$ $\sigma+\tau, \rho+\sigma-\tau, \rho-\sigma+\tau$ is an odd integer, or

$B$. the numbers $\rho$ or $-\rho$ and $\sigma$ or $-\sigma$ and $\tau$ or $-\tau$ belong (in an arbitrary order) to some of appropriate fifteen families forming the so-called Schwarz's Table 1.

\section{References}

1. Elmandouh, A.A.: On the integrability of 2D Hamiltonian systems with variable Gaussian curvature. Nonlinear Dynam. 93, 933-943 (2018)

2. Bialy, M., Mironov, A.E.: Integrable geodesic flows on 2torus: formal solutions and variational principle. J. Geom. Phys. 87, 39-47 (2015)

3. A. V. Bolsinov and B. Jovanović. Integrable geodesic flows on Riemannian manifolds: construction and obstructions. In Contemporary geometry and related topics, pages 57-103. World Sci. Publ., River Edge, NJ, 2004

4. Dullin, H.R., Matveev, V.S.: A new integrable system on the sphere. Math. Res. Lett. 11(5-6), 715-722 (2004)

5. Kiyohara, K.: Two-dimensional geodesic flows having first integrals of higher degree. Math. Ann. 320(3), 487-505 (2001)

6. Kiyohara, K.: Periodic geodesic flows and integrable geodesic flows. Sūgaku 56(1), 88-98 (2004)

7. Matveev, V.S., Shevchishin, V.V.: Two-dimensional superintegrable metrics with one linear and one cubic integral. J. Geom. Phys. 61(8), 1353-1377 (2011)

8. Kozlov, V.V., Denisova, N.V.: Polynomial integrals of geodesic flows on a two-dimensional torus. Mat. Sb. 185(12), 49-64 (1994)

9. Galajinsky, A., Lechtenfeld, O.: On two-dimensional integrable models with a cubic or quartic integral of motion. J. High Energy 2013(9), 1-12 (2013)

10. Valent, G., Duval, C., Shevchishin, V.: Explicit metrics for a class of two-dimensional cubically superintegrable systems. J. Geom. Phys. 87, 461-481 (2015) 
11. Kruglikov, B.S., Matveev, V.S.: Nonexistence of an integral of the 6th degree in momenta for the zipoy-voorhees metric. Phys. Rev. D 85, 124057 (2012)

12. Yehia, H.M.: On certain two-dimensional conservative mechanical systems with a cubic second integral. J. Phys. A 35(44), 9469-9487 (2002)

13. Yehia, H.M.: Two-dimensional conservative mechanical systems with quartic second integral. Regul. Chaotic Dyn. 11(1), 103-122 (2006)

14. Valent, G.: On a class of integrable systems with a cubic first integral. Comm. in Math. Phys. 299(3), 631-649 (2010)

15. Valent, G.: On a class of integrable systems with a quartic first integral. Regul. Chaotic Dyn. 18(4), 394-424 (2013)

16. Hadeler, K.P., Selivanova, E.N.: On the case of Kovalevskaya and new examples of integrable conservative systems on $\mathbb{S}^{2}$. Regul. Chaotic Dyn 4(3), 45-52 (1999)

17. Selivanova, E.N.: New families of conservative systems on $\mathbb{S}^{2}$ possessing an integral of fourth degree in momenta. Ann. Global Anal. Geom. 17(3), 201-219 (1999)

18. Ballesteros, Á., Blasco, A., Herranz, F.J., Musso, F.: An integrable Hénon-Heiles system on the sphere and the hyperbolic plane. Nonlinearity 28(11), 3789-3801 (2015)

19. Á. Ballesteros, F. J. Herranz, S.1 Kuru, Javier Negro.: The anisotropic oscillator on curved spaces: a new exactly solvable model. Ann. Phys. 373:399-423, 2016

20. Maciejewski, A.J., Szumiński, W., Przybylska, M.: Note on integrability of certain homogeneous Hamiltonian systems in $2 \mathrm{D}$ constant curvature spaces. Phys. Lett. A 381(7), 725732 (2017)

21. Szumiński, W.: Integrability analysis of natural Hamiltonian systems in curved spaces. Commun. Nonlinear Sci. Numer. Simulat. 64, 246-255 (2018)

22. J. J. Morales-Ruiz. Differential Galois theory and nonintegrability of Hamiltonian systems. Progress in Mathematics, Birkhauser Verlag, Basel, 1999

23. D. Boucher and J. A. Weil. Application of J.-J. Morales J.-P. Ramis.:theorem to test the non-complete integrability of the planar three-body problem. IRMA Lect. Math. Theor. Phys., 3:163-177, 2003

24. Maciejewski, A.J., Przybylska, M.: Non-integrability of the three-body problem. Celestial Mech. Dynam. Astronom. 110(1), 17-300 (2011)

25. Szumiński, W.: Integrability analysis of chaotic and hyperchaotic finance systems. Nonlinear Dynam. 94(1), 443-459 (2018)

26. Szumiński, W.: On certain integrable and superintegrable weight-homogeneous Hamiltonian systems. Commun. Nonlinear Sci. Numer. Simulat. 67, 600-616 (2018)

27. Maciejewski, A.J., Szumiński, W.: Non-integrability of the semiclassical Jaynes-Cummings models without the rotating-wave approximation. Appl. Math. Lett. 82, 132139 (2018)
28. Maciejewski, A.J., Przybylska, M., Szumiński, W.: Anisotropic Kepler and anisotropic two fixed centres problems. Celestial Mech. Dynam. Astronom. 127(2), 163-184 (2017)

29. Szumiński, W., Maciejewski, A.J., Przybylska, M.: Note on integrability of certain homogeneous Hamiltonian systems. Phys. Lett. A 379(45-46), 2970-2976 (2015)

30. Stachowiak, T., Szumiński, W.: Non-integrability of restricted double pendula. Phys. Lett. A 379(47-48), 30173024 (2015)

31. Maciejewski, A.J., Przybylska, M., Simpson, L., Szumiński, W.: Non-integrability of the dumbbell and point mass problem. Celestial Mech. Dynam. Astronom. 117(3), 315-330 (2013)

32. Przybylska, M., Szumiński, W.: Non-integrability of flail triple pendulum. Chaos Soliton. Fract. 53, 60-74 (2013)

33. Szumiński, W., Przybylska, M.: Differential Galois integrability obstructions for nonlinear three-dimensional differential systems. Chaos 30, 013135 (2020)

34. J. J. Morales-Ruiz and J.-P. Ramis.: Integrability of dynamical systems through differential Galois theory: a practical guide. In Differential algebra, complex analysis and orthogonal polynomials, volume 509 of Contemp. Math., pages 143-220. Amer. Math. Soc., Providence, RI, 2010

35. Kovacic, J.J.: An algorithm for solving second order linear homogeneous differential equations. J. Symb. Comput. 2(1), 461-481 (1986)

36. Kimura, T.: On Riemann's equations which are solvable by quadratures. Funkcial. Ekvac 12, 269-281 (1969)

37. Elmandouh, A.A.: First integrals of motion for two dimensional weight-homogeneous Hamiltonian systems in curved spaces. Commun. Nonlinear Sci. Numer. Simulat. 75, 220 235 (2019)

38. Maciejewski, A.J., Przybylska, M.: Integrability of Hamiltonian systems with algebraic potentials. Phys. Lett. A 380(12), 76-82 (2016)

39. Combot, T.: A note on algebraic potentials and MoralesRamis theory. Celest. Mech. Dyn. Astr. 115, 397-404 (2012)

40. Whittaker, E.T., Watson, G.N.: A Course of Modern Analysis. Cambridge University Press, London (1935)

41. G. Kristensson.: Second Order Differential Equations, Special Functions and Their Classification. Springer, New York , 2010

Publisher's Note Springer Nature remains neutral with regard to jurisdictional claims in published maps and institutional affiliations. 